\title{
In vivo early diagnosis of gastric dysplasia using narrow-band image-guided Raman endoscopy
}

\author{
Zhiwei Huang \\ Mads Sylvest Bergholt \\ Wei Zheng \\ Kan Lin \\ National University of Singapore \\ Department of Bioengineering \\ Faculty of Engineering \\ Optical Bioimaging Laboratory \\ Singapore 117576
}

\section{Khek Yu Ho}

National University of Singapore and

National University Hospital

Yong Loo Lin School of Medicine

Department of Medicine

Singapore 119260

\author{
Ming Teh \\ National University of Singapore \\ and \\ National University Hospital \\ Yong Loo Lin School of Medicine \\ Department of Pathology \\ Singapore 119074
}

\section{Khay Guan Yeoh}

National University of Singapore and

National University Hospita

Yong Loo Lin School of Medicine

Department of Medicine

Singapore 119260

\begin{abstract}
We first report on the implementation of a novel narrowband image-guided Raman endoscopy technique for in vivo diagnosis of gastric dysplasia. High-quality in vivo Raman spectra can be acquired from normal and dysplastic gastric mucosal tissue within $0.5 \mathrm{sec}$ under narrow-band image (NBI) guidance at gastroscopy. Significant differences are observed in in vivo Raman spectra between normal $(n=54)$ and dysplastic $(n=18)$ gastric tissue from 30 gastric patients, particularly in the spectral ranges of 825 to 950,1000 to 1100, 1250 to 1500 , and 1600 to $1800 \mathrm{~cm}^{-1}$, which primarily contain signals related to proteins, nucleic acids, and lipids. The multivariate analysis [i.e., principal components analysis (PCA) and linear discriminant analysis (LDA)], together with the leave-one tissue siteout, cross validation on in vivo gastric Raman spectra yields a diagnostic sensitivity of $94.4 \%(17 / 18)$ and specificity of $96.3 \%(52 / 54)$ for distinction of gastric dysplastic tissue. This study suggests that narrowband image-guided Raman endoscopy associated with PCA-LDA diagnostic algorithms has potential for the noninvasive, in vivo early diagnosis and detection of gastric precancer during clinical gastroscopic examination. () 2010 Society of Photo-Optical Instrumentation Engineers. [DOI: 10.1117/1.3420115]
\end{abstract}

Keywords: gastric dysplasia; in vivo diagnosis; Raman endoscopy; narrow-band imaging; gastroscopy; precancer.

Paper 10001LRR received Jan. 4, 2010; revised manuscript received Mar. 27, 2010; accepted for publication Apr. 1, 2010; published online Jun. 1, 2010.

\section{Introduction}

Gastric cancer is the second leading cause of cancerassociated death, accounting for approximately 600,000 annual deaths worldwide. ${ }^{1}$ Early diagnosis and localization with appropriate curative treatments (e.g., endoscopic submucosal dissection and gastrectomy) is critical to decreasing mortality. ${ }^{2}$ However, identification of early cancer and precancer can be difficult, as conventional white-light reflectance (WLR) endoscopy heavily relies on visual identification of morphological tissue changes. Thus, subtle changes of gastric precancer (i.e., dysplasia) and early cancer may not be apparent, limiting diagnostic accuracy. Positive endoscopic biopsy is the standard criterion for gastric precancer and cancer diagnosis, but it is invasive and impractical for screening highrisk patients who may have multiple suspicious lesions. ${ }^{1,2}$ Very recently, narrow-band imaging (NBI) that enhances visualization of irregular mucosal and vascular patterns has

Address all correspondence to: Dr. Zhiwei Huang, Optical Bioimaging Laboratory, Department of Bioengineering, Faculty of Engineering, National University of Singapore, 9, Engineering Drive 1, Singapore 117576. Tel: (65) 6516-8856; Fax: (65) 6872-3069; E-mail: biehzw@nus.edu.sg shown promise for improving in vivo diagnosis of intraepithelial neoplastic lesions in gastric tissue. ${ }^{3}$ Although the NBI technique provides good detection sensitivities, this wide-field endoscopic imaging modality still suffers from moderate diagnostic specificity due to the deficiency of revealing specific biochemical information of tissue. Hence, a noninvasive optical diagnostic technique providing a direct assessment of biochemical information of suspicious lesions in vivo will represent a significant advance in endoscopic detection of precancer and early cancer in gastric tissue.

In the past decade, Raman spectroscopy, which measures inelastic light scattering processes and is capable of providing spectroscopic fingerprints of specific molecular structures and compositions of biological tissues, has been comprehensively investigated for precancer and cancer diagnosis and evaluation in a number of organs, including the stomach. ${ }^{4-14}$ These investigations show that specific spectral features of Raman spectra could be used to correlate with the molecular and structural changes of tissue associated with neoplastic trans-

1083-3668/2010/15(3)/037017/5/\$25.00 @ 2010 SPIE 
formation. Diagnostic sensitivities of $\sim 85$ to $95 \%$ and specificities of $\sim 90$ to $98 \%$ have been reported for differentiation between normal and pathological (e.g., dysplasia, adenocarcinoma) gastric tissues in vitro using Raman spectroscopic technique. ${ }^{12-14}$ However, to date, in vivo Raman studies for early diagnosis of gastric precancer and cancer have not been reported. In vivo Raman endoscopic applications have been limited not only by the difficulty of capturing inherently weak tissue Raman signals, but also by the relatively high speed of spectral acquisitions required in clinical settings. ${ }^{4-6}$ The fabrication of a millimeter-scaled or even smaller flexible fiber optic Raman probe with abilities of efficient fiber fluorescence/Raman background rejections while having high tissue Raman signal collection efficiency renders another technical challenge for clinical Raman endoscopic examinations. ${ }^{6,8,15}$ Very recently, we have successfully developed a high throughput Raman endoscopy system integrated with trimodal wide-field endoscopic imaging for rapid in vivo gastric tissue Raman measurements at gastroscopy. ${ }^{16}$ With the unique image-guided Raman endoscopy technique developed, we aim, in this work, to evaluate the clinical utility of Raman spectroscopy for in vivo diagnosis and detection of gastric dysplasia during clinical gastroscopic examination.

\section{Materials and Methods}

The Raman endoscopy system developed ${ }^{16}$ consists of a spectrum-stabilized 785-nm diode laser (maximum output: 300 mW, B\&W TEK Incorporated, Newark, Delaware), a transmissive imaging spectrograph (Holospec f/1.8, Kaiser Optical Systems, Ann Arbor, Michigan), a liquid nitrogencooled, near-infrared (NIR)-optimized, back-illuminated deep depletion charge-coupled device (CCD) camera (Spec-10: 400BR/LN, Princeton Instruments, Trenton, New Jersey), and a specially designed Raman endoscopic probe for both laser light delivery and in vivo tissue Raman signal collection. The 1.8-mm fiber optic Raman endoscopic probe, which can fit into the biopsy channel of medical endoscopes, consists of 32 Raman collection fibers (core diameter of $200 \mu \mathrm{m}$ ) surrounding the central laser light delivery fiber (core diameter of $200 \mu \mathrm{m}$ ), with two stages of optical filtering incorporated at the proximal and distal ends of the probe for maximizing the collection of tissue Raman signals while reducing the interference of Rayleigh scattered light, fiber fluorescence, and silica Raman signals. ${ }^{16}$ A personal computer controls the system using a custom-designed software that triggers real-time data acquisition and online data analysis (e.g., CCD dark-noise subtraction, wavelength calibration, system spectral response calibration, signal saturation detection, cosmic ray rejection, tissue autofluorescence background subtraction, etc.). The system acquires in vivo tissue Raman spectra in the wave number range of 800 to $1800 \mathrm{~cm}^{-1}$, and the spectral resolution of the system is $9 \mathrm{~cm}^{-1}$. Each spectrum was acquired within $0.5 \mathrm{sec}$ using the $785-\mathrm{nm}$ laser light irradiance of $1.5 \mathrm{~W} / \mathrm{cm}^{2}$, which is less than the American National Standards Institute (ANSI) maximum permissible skin exposure limit set out for a 785-nm laser beam. ${ }^{17}$ Our further calculations based on the finite difference thermal model ${ }^{18,19}$ and the optical properties of stomach tissue ${ }^{20}$ indicate that even without consideration of other cooling effects (e.g., perfusion and evaporation in tissue), the maximum tissue temperature rise is only about $0.15^{\circ} \mathrm{C}$ after $1 \mathrm{~min}$ of $785-\mathrm{nm}$ laser radiation with an incident power of $30 \mathrm{~mW}$ on a tissue spot size of $200 \mu \mathrm{m}$ during tissue Raman measurements. This temperature rise estimated is far below the level to generate cytotoxicity in tissue and cells, ${ }^{21}$ suggesting that the laser power density used in this study is safe for in vivo tissue Raman measurements. Note that the raw spectra acquired from in vivo gastric tissue represent a combination of intense tissue autofluorescence, weak tissue Raman scattering signals, and noise. These raw spectra are preprocessed by a first-order Savitsky-Golay filter (window width of 3 pixels, corresponding to the system spectral resolution) to reduce noise. A fifthorder polynomial ${ }^{5}$ is found to be optimal for fitting the broad autofluorescence background in the noise-smoothed spectrum, and this polynomial is then subtracted from the raw spectrum to yield the tissue Raman spectrum alone. Each backgroundsubtracted Raman spectrum is also normalized to the integrated area under the curve from 800 to $1800 \mathrm{~cm}^{-1}$, enabling better comparison of the spectral shapes and relative peak intensities among different gastric tissues. ${ }^{5}$ All of the spectra preprocessing is completed online and the Raman spectrum can be displayed in real time during clinical Raman measurements at gastroscopy. The trimodal endoscope imaging system primarily comprises a $300-\mathrm{W}$ dedicated short-arc xenon light source, a gastrointestinal (GI) video-endoscope (GIFFQ260Z, Olympus), and a video system processor (CV260SL, Olympus) for white-light reflectance (WLR) imaging, autofluorescence imaging (AFI), and narrow-band imaging (NBI) during gastroscopic examination. Both the wide-field endoscopic image (WLR/NBI/AFI) and the point-wise in vivo Raman spectra of the tissue imaged can be simultaneously acquired, stored, and displayed in real time on a color video monitor and computer screen, respectively.

In this work, all patients signed an informed consent permitting the in vivo Raman endoscopic measurements of gastric tissue in the Endoscope Centre at the National University Hospital (NUH), Singapore. This study was approved by the Institutional Review Board (IRB) of the National Healthcare Group (NHG) of Singapore. We have acquired in vivo Raman spectra [Fig. 1(a)] of 72 gastric mucosal tissue sites in 30 gastric patients (16 men and 14 women, with a median age of 67) under the guidance of wide-field endoscopic imaging [e.g., NBI and WLR imaging in Fig. 1(b)] during gastroscopic examination. The Raman endopscopic probe was placed in gentle contact with the gastric mucosa surface, and the positioning against the tissue sites was verified on the endoscopy monitor by the endoscopists in charge during gastroscopic examinations. Immediately after all Raman acquisitions, the biopsy samples were taken from the tissue sites measured (with suction markings) and fixed in $10 \%$ formalin solution for histopathological examinations by a senior gastrointestinal pathologist. For the assessment of diagnostic sensitivity and specificity of Raman endoscopy for gastric tissue classification, histopathological results served as the golden standard.

\section{Results and Discussion}

Figure 1(a) shows the in vivo mean Raman spectra \pm 1 standard deviations (SD) and the corresponding Raman difference spectrum of normal $(n=54)$ and dysplastic $(n=18)$ gastric tissue. The representative NBI and WLR image of dysplastic 


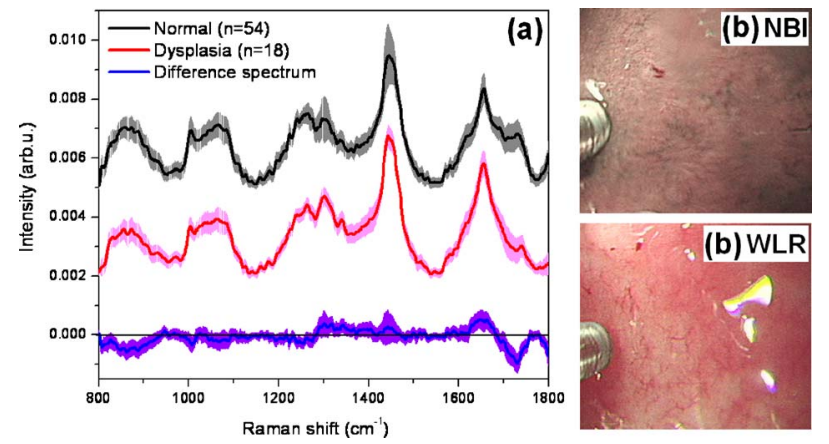

Fig. 1 In vivo mean Raman spectra \pm 1 standard deviations (SD) of normal $(n=54)$ and dysplastic $(n=18)$ gastric tissue. The difference spectrum (i.e., the mean Raman spectrum of dysplastic tissue minus the mean Raman spectrum of normal tissue) reveals the Raman spectral changes, particularly in the regions of 825 to 950, 1000 to 1100 , 1250 to 1500 , and 1600 to $1800 \mathrm{~cm}^{-1}$, associated with dysplastic transformation. Note that in vivo Raman spectra of both normal and dysplastic gastric tissues are vertically shifted for better visualization. The shaded areas in tissue Raman spectra stand for the respective standard deviations. (b) Narrow-band image (NBI) and white-light reflectance (WLR) image of dysplastic gastric lesions in the antrum acquired during clinical gastroscopy. NBI enhances the observation of the irregular mucosal glandular patterns and vascular patterns of dysplastic gastric mucosal lesions.

gastric tissue acquired under Raman endoscopic measurements are shown in Fig. 1(b). All in vivo tissue Raman spectra are acquired within 0.1 to $0.5 \mathrm{sec}$ (depending on the autofluorescence background level of different gastric tissues) with the 785-nm light irradiance power of $1.5 \mathrm{~W} / \mathrm{cm}^{2}$. Prominent Raman bands are observed in both normal and dysplastic gastric tissue at the following peak positions with tentative biochemical assignments: $:^{4-7,9-14} 875 \mathrm{~cm}^{-1}[v(\mathrm{C}=\mathrm{C})$ hydroxyproline $], \quad 1004 \mathrm{~cm}^{-1} \quad[v(\mathrm{C}-\mathrm{C})$ ring breathing of phenylalanine], $1078 \mathrm{~cm}^{-1}[v(\mathrm{CC})$ or $v(\mathrm{CO})$ of phospholipids], $1265 \mathrm{~cm}^{-1}$ [amide III $v(\mathrm{CN})$ and $\delta(\mathrm{NH})$ of proteins], $1302 \mathrm{~cm}^{-1}\left(\mathrm{CH}_{2} \mathrm{CH}_{3}\right.$ twisting of proteins and nucleic acids), $1450 \mathrm{~cm}^{-1}\left[\delta\left(\mathrm{CH}_{2}\right)\right.$ of proteins and lipids $], 1655 \mathrm{~cm}^{-1}$ [amide I $v(\mathrm{C}=\mathrm{O})$ of proteins], and $1745 \mathrm{~cm}^{-1}[v(\mathrm{C}=\mathrm{O})$ of phospholipids]. The difference spectrum [Fig. 1(a)] reveals the changes of relative percentages of distinctive biomolecules in dysplastic tissue, particularly in the spectral ranges of 825 to 950,1000 to 1100,1250 to 1500 , and 1600 to $1800 \mathrm{~cm}^{-1}$, which contain signals related to proteins, nucleic acids, and lipids, respectively. Dysplasia tissues show lower intensities at 875, 1004, 1078, 1210, and $1745 \mathrm{~cm}^{-1}$, while higher intensities at $1265,1302,1450$, and $1655 \mathrm{~cm}^{-1}$ compared with normal tissue. This indicates that there is an increase or decrease in the percentage of a certain type of biomolecules relative to the total Raman-active constituents in gastric tissue associated with dysplastic transformation. More specifically, the decrease in Raman signals at $875 \mathrm{~cm}^{-1}$ (hydroxyproline of collagen) may reflect cytoplasmic mucin depletion and the elevated concentration of metalloproteinase that cleaves collagen in the stroma layer of gastric dysplasia tissue. ${ }^{13,14,22,23}$ In addition, the thickening of the epithelium associated with dysplastic progression can attenuate the excitation laser power and also obscure the collagen Raman emission from the deep basal membrane, ${ }^{24,25}$ thereby resulting in

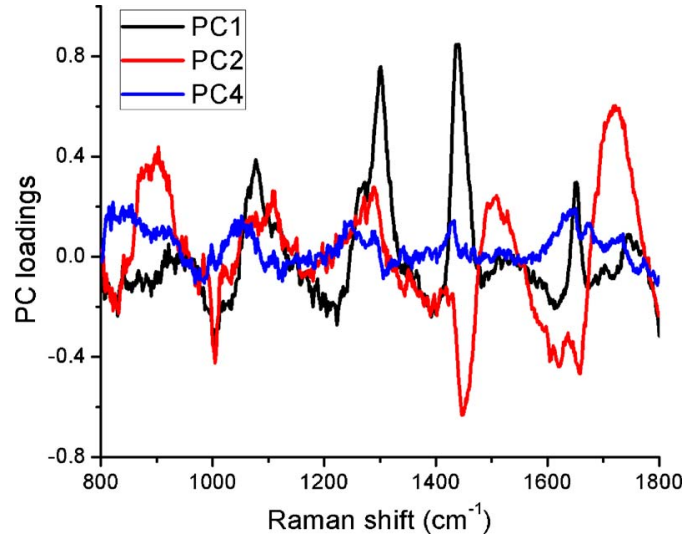

Fig. 2 The three significant principal components (PCs) (PC1 $\sim 52.6 \%$, PC2 $13.2 \%$, and PC 4 4.7\%) accounting for $\sim 70.5 \%$ of the total variance calculated from in vivo gastric Raman spectra.

an overall decrease of Raman intensity at $875 \mathrm{~cm}^{-1}$ from dysplasia tissue. The increase of Raman signals attributed to proteins (e.g., 1265, 1302, 1450, and $1655 \mathrm{~cm}^{-1}$ ) in gastric dysplasia may be associated with an increase in the metabolic activities (e.g., increased mitotic activities that include enzymes, hormones, etc.) as well as the increased hyperchromatism and the nucleic acids-to-cytoplasm ratio of dysplastic cells. $^{23,24}$ The decrease in Raman signals in the region $1745 \mathrm{~cm}^{-1}$ suggests a reduction of phospholipids in dysplastic tissue that also has been found in other epithelial tissues with malignancies. ${ }^{5,10}$ Further, there are also obvious changes of Raman peak shifts and bandwidths in the spectral ranges of 1200 to $1500 \mathrm{~cm}^{-1}$ and 1600 to $1800 \mathrm{~cm}^{-1}$, which are related to the amide III and amide I of proteins, $\mathrm{CH}_{3} \mathrm{CH}_{2}$ twisting of proteins/nucleic acids, and $\mathrm{C}=\mathrm{O}$ stretching of phospholipids for dysplasia. The current results of in vivo gastric tissue Raman spectra are also similar to our previous observations of in vitro gastric tissue Raman spectra. ${ }^{12-14}$ Therefore, the distinctive differences in in vivo Raman spectra between normal and dysplastic tissue confirm the potential role of Raman endoscopy for in vivo gastric precancer diagnosis at the molecular level.

We have employed the multivariate statistical technique [i.e., principal components analysis (PCA) and linear discriminant analysis (LDA)] by utilizing the entire Raman spectra to determine the most diagnostically significant Raman features for gastric tissue classification. The unpaired, twosided Student's t-test on the obtained principal components (PCs) shows that the three PCs (PC1, PC2, and PC4) (Fig. 2) accounting for $\sim 70.5 \%$ of the total variance contain the most diagnostically significant Raman features $(p<0.05)$ for discriminating dysplasia from normal gastric tissue. The first PC accounts for the largest variance $(\sim 52.6 \%$ of the total variance), whereas the successive PCs describe the spectral features that contribute progressively smaller variances (e.g., PC2 13.2\%, PC4 4.7\%). Some PC features, such as the peaks, troughs, and spectral shapes in Fig. 2, are similar to those of tissue Raman spectra (Fig. 1).

All three significant PCs were loaded into the LDA model to develop effective diagnostic algorithms for gastric tissue diagnosis. Figure 3 shows the classification results of in vivo 


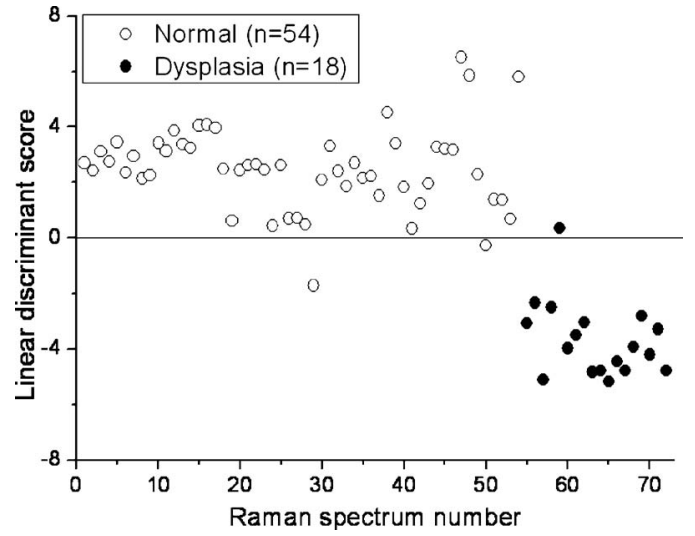

Fig. 3 Scatter plot of the linear discriminant scores belonging to normal and dysplasia gastric tissue using the PCA-LDA algorithms, together with the leave-one tissue site-out, cross-validation method. The separate line yields a diagnostic sensitivity of $94.4 \%(17 / 18)$ and specificity of $96.3 \%(52 / 54)$ for distinguishing dysplasia from normal gastric tissue in vivo.

Raman spectra of normal and precancer gastric tissue using PCA-LDA together with the leave-one tissue site-out, crossvalidation techniques. The PCA-LDA algorithm based on in vivo gastric Raman spectra provides a diagnostic sensitivity of $94.4 \%(17 / 18)$ and specificity of $96.3 \%(52 / 54)$ for distinguishing dysplasia from normal gastric tissue in vivo. To further evaluate the performance of the PCA-LDA-based diagnostic algorithms derived from all three significant PCs of in vivo gastric Raman datasets, the receiver operating characteristic (ROC) curve (Fig. 4) is also generated from the scatter plot in Fig. 3 at different threshold levels, displaying the discrimination results using PCA-LDA diagnostic algorithms together with the leave-one tissue site-out, cross-validation method. The integration area under the ROC curve is 0.997 , further demonstrating the diagnostic efficacy of Raman endo-

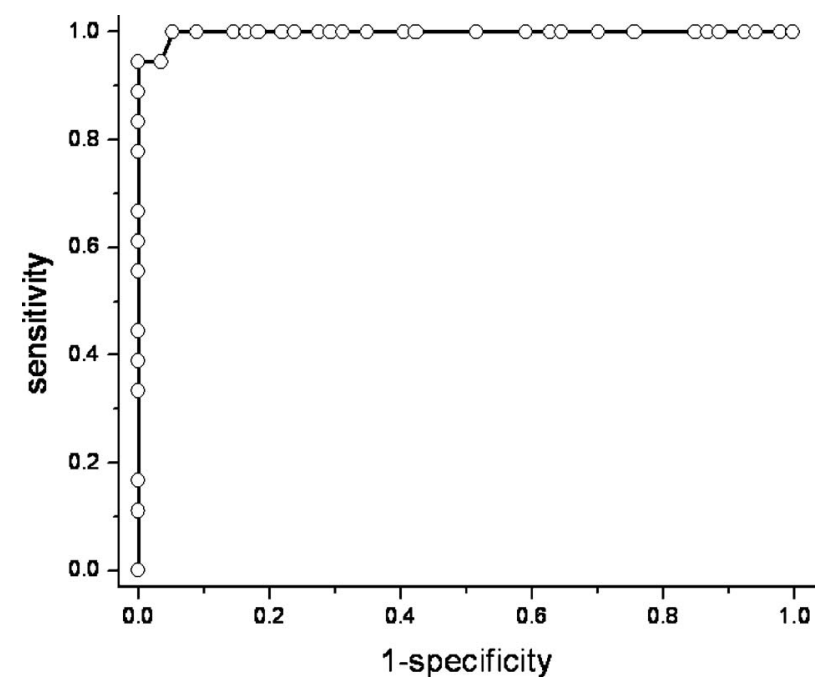

Fig. 4 Receiver operating characteristic (ROC) curve of discrimination results of in vivo Raman spectra of normal and dysplastic gastric tissue using the PCA-LDA algorithms, together with the leave-one tissue site-out, cross-validation method. scopy for in vivo diagnosis of gastric precancer during clinical gastroscopic examination.

In summary, we have acquired, for the first time, highquality in vivo Raman spectra from normal and dysplastic gastric tissue within $0.5 \mathrm{sec}$ under narrow-band imaging guidance during clinical gastroscopy. We have observed the significant differences in in vivo Raman spectra between normal and dysplastic gastric tissue. Good differentiation between normal and dysplastic gastric tissues can be achieved using PCA-LDA diagnostic algorithms, indicating the potential of Raman endoscopy for in vivo diagnosis of gastric precancer. Currently, we are conducting in vivo Raman measurements on a larger series of gastric patients at National University Hospital, Singapore, to further evaluate the clinical merits of Raman endoscopy techniques for prospective prediction of gastric precancer and early cancer in vivo during gastroscopic examination.

\section{Acknowledgments}

This research was supported by the National Medical Research Council, the Biomedical Research Council, and the Faculty Research Fund from the National University of Singapore.

\section{References}

1. B. J. Dicken, D. L. Bigam, C. Cass, J. R. Mackey, A. A. Joy, and S. M. Hamilton, "Gastric adenocarcinoma: review and considerations for future directions," Ann. Surg. 241, 27-39 (2005).

2. C. J. Clark, R. C. Thirlby, V. Jr. Picozzi, D. B. Schembre, F. P. Cummings, and E. Lin, "Current problems in surgery: gastric cancer," Curr. Probl Surg. 43, 566-570 (2006).

3. A. J. Overhiser and P. Sharma, "Advances in endoscopic imaging: narrow band imaging," Rev. Gastroenterol. Disord. 8, 186-193 (2008).

4. N. Stone, P. Stavroulaki, C. Kendall, M. Birchall, and H. Barr, "Raman spectroscopy for early detection of laryngeal malignancy: preliminary results," Laryngoscope 110, 1756-1763 (2000).

5. Z. Huang, A. McWilliams, H. Lui, D. I. McLean, S. Lam, and H. Zeng, "Near-infrared Raman spectroscopy for optical diagnosis of lung cancer," Int. J. Cancer 107, 1047-1052 (2003).

6. M. G. Shim, L. M. Song, N. E. Marcon, and B. C. Wilson, "In vivo near-infrared Raman spectroscopy: demonstration of feasibility during clinical gastrointestinal endoscopy," Photochem. Photobiol. 72, 146-150 (2000).

7. E. Widjaja, W. Zheng, and Z. Huang, "Classification of colonic tissues using near-infrared Raman spectroscopy and support vector machines," Int. J. Oncol. 32, 653-662 (2008).

8. M. A. Short, S. Lam, A. McWilliams, J. Zhao, H. Lui, and H. Zeng, "Development and preliminary results of an endoscopic Raman probe for potential in vivo diagnosis of lung cancers," Opt. Lett. 33, 711713 (2008).

9. D. P. Lau, Z. Huang, H. Lui, C. S. Man, K. Berean, M. D. Morrison, and H. Zeng, "Raman spectroscopy for optical diagnosis in normal and cancerous tissue of the nasopharynx-preliminary findings," $\mathrm{La}$ sers Surg. Med. 32, 210-214 (2003).

10. S. K. Teh, W. Zheng, D. P. Lau, and Z. Huang, "Spectroscopic diagnosis of laryngeal carcinoma using near-infrared Raman spectroscopy and random recursive partitioning ensemble techniques," Analyst (Cambridge, U.K.) 134, 1232-1239 (2009).

11. A. Nijssen, T. C. Bakker, F. Heule, P. J. Caspers, D. P. Hayes, M. H. Neumann, and G. J. Puppels, "Discriminating basal cell carcinoma from its surrounding tissue by Raman spectroscopy," J. Invest. Dermatol. 119, 64-69 (2002).

12. S. K. Teh, W. Zheng, K. Y. Ho, M. Teh, K. G. Yeoh, and Z. Huang, "Diagnosis of gastric cancer using near-infrared Raman spectroscopy and classification and regression tree techniques," J. Biomed. Opt. 13, 034013 (2008) 
13. S. K. Teh, W. Zheng, K. Y. Ho, M. Teh, K. G. Yeoh, and Z. Huang, "Near-infrared Raman spectroscopy for gastric precancer diagnosis," J. Raman Spectrosc. 40, 908-914 (2009).

14. S. K. Teh, W. Zheng, K. Y. Ho, M. Teh, K. G. Yeoh, and Z. Huang, "Diagnostic potential of near-infrared Raman spectroscopy in the stomach: differentiating dysplasia from normal tissue," Br. J. Cancer 98, 457-465 (2008).

15. Y. Hattori, Y. Komachi, T. Asakura, T. Shimosegawa, G. Kanai, H. Tashiro, and H. Sato, "In vivo Raman study of the living rat esophagus and stomach using a micro-Raman probe under an endoscope," Appl. Spectrosc. 61, 579-584 (2007).

16. Z. Huang, S. K. Teh, W. Zheng, J. Mo, K. Lin, X. Shao, K. Y. Ho, M. Teh, and K. G. Yeoh, "Integrated Raman spectroscopy and trimodal wide-field imaging techniques for real-time in vivo tissue Raman measurements at endoscopy," Opt. Lett. 34, 758-760 (2009).

17. "American National Standard for the Safe Use of Lasers," ANSI Standard 2136.1-1986, American National Standards Institute, Washington, D.C. (1986).

18. F. P. Incropera and D. P. D. Witt, Fundamentals of Heat and Mass Transfer, John Wiley and Sons, New York (1990).

19. J. H. Torres, M. Motamedi, J. A. Pearce, and A. J. Welch, "Experimental evaluation of mathematical models for predicting the therma response of tissue to laser irradiation," Appl. Opt. 32, 597-606 (1993).
20. A. N. Bashkatov, E. A. Genina, V. I. Kochubey, A. A. Gavrilova, S V. Kapralov, V. A. Grishaev, and V. V. Tuchin, "Optical properties of human stomach mucosa in the spectral range from 400 to $2000 \mathrm{~nm}$ : Prognosis for gastroenterology," Med. Laser Appl. 22, 95-104 (2007).

21. S. Thomsen, "Pathologic analysis of photothermal and photomechanical effects of laser-tissue interactions," Photochem. Photobiol. 53, 825-835 (1991).

22. P. Correa, "A human model of gastric carcinogenisis," Cancer Res. 48, 3554-3560 (1988)

23. I. Georgakoudi, B. C. Jacobson, M. G. Müller, E. E. Sheets, K. Badizadegan, D. L. Carr Locke, C. P. Crum, C. W. Boone, R. R. Dasari, J. Van Dam, and M. S. Feld, "NAD(P)H and collagen as an in vivo quantitative fluorescence biomarkers of epithelial precancerous changes," Cancer Res. 62, 682-687 (2002).

24. J. H. Hughes, C. J. Leigh, S. S. Raab, S. Y. Hook, M. B. Cohen, and M. J. Suhrland, "Cytologic criteria for the brush diagnosis of gastric adenocarcinoma," Cancer 84, 289-294 (1998).

25. Z. Huang, W. Zheng, S. Xie, R. Chen, H. Zeng, D. I. McLean, and H. Lui, "Laser-induced autofluorescence microscopy of normal and tumor human colonic tissue," Int. J. Oncol. 24, 59-64 (2004). 